This item was submitted to Loughborough's Research Repository by the author.

Items in Figshare are protected by copyright, with all rights reserved, unless otherwise indicated.

\title{
A new algorithm for calculating perceived colour difference of images
}

PLEASE CITE THE PUBLISHED VERSION

PUBLISHER

(C) Maney

LICENCE

CC BY-NC-ND 4.0

\section{REPOSITORY RECORD}

Hong, Guowei, and M. Ronnier Luo. 2019. "A New Algorithm for Calculating Perceived Colour Difference of Images”. figshare. https://hdl.handle.net/2134/2127. 
Paper Title: A new algorithm for calculating perceived colour difference of images

Authors: Guowei Hong and Ronnier Luo*

Affiliation: Applied Vision Research Centre, Ergonomics and Safety Research Institute, Loughborough University, UK

*Department of Colour and Polymer Chemistry, University of Leeds, UK

\section{Address for manuscript correspondence:}

Dr. Guowei Hong

Applied Vision Research Centre

Ergonomics and Safety Research Institute

Loughborough University

Loughborough LE11 3UZ

UK

Tel: +44-1332-593104

Fax: +44-1332-593103

Email: g.hong@derby.ac.uk 


\begin{abstract}
Faithful colour reproduction of digital images requires a reliable measure to compare such images in order to evaluate the reproduction performance. The conventional methods attempt to apply the CIE Colorimetry based colour difference equations, such as CIELAB, CMC, CIE94 and CIEDE2000, to complex images on a pixel-by-pixel basis, and calculates the overall colour difference as the averaged difference of each pixel in the image. This method is simple and straightforward but often does not represent the colour difference perceived by human visual system. This paper proposes a new algorithm for calculating the overall colour difference between a reproduced image and its original. The results obtained show that this new metric provides a quantitative measure that more closely corresponds to the colour difference perceived by human visual system.
\end{abstract}

Key words: colour difference, colour reproduction, colour appearance, colour image processing, colour imaging 


\section{Introduction}

With the rapid growth of digital colour imaging, faithful colour reproduction of digital images is becoming a major challenge today. The ultimate goal is to allow an automatic control of all imaging devices and to move digital colour images from one media to another without losing the visual appearance of the original image. After a digital image is reproduced, a measure for calculating the colour difference between the reproduced and the original images is required to evaluate the reproduction performance. This ability to quantitatively measure the colour difference between two digital colour images is necessary to allow for an objective evaluation of the performance of the reproduction system and subsequently an optimised reproduction. Obviously this measurement should correspond to the subjective assessment of the human visual system. Indeed, if the computed image-based colour difference can truly represent human visual assessment, those time-consuming and laborious psychophysical experiments on comparing reproduced images can be avoided. The need for this kind of image-based colour difference algorithm [1-3] has been widely recognised and has been brought to the forefront with the formation of the CIE Technical Committee 8-2 [4].

Current colour difference formulae such as CIELAB [5], CMC [6], CIE94 [7], and CIEDE2000 [8] are all based on the CIE Colorimetry. They were derived from psychophysical experiments on assessing small colour difference using large size uniform surface patches under uniform grey backgrounds. Because of their successful application for calculating colour difference for large size uniform surface patches and the lack of image-based colour difference formula, these equations were often adopted for calculating 
colour difference for complex images. The current typical method attempts to apply these colour difference equations to images on a pixel-by-pixel basis, and calculates the overall colour difference as the averaged difference of each pixel in the image. Song and Luo [9] have recently adopted this approach and compared the performances of all above mentioned colour difference formulae on a set of complex images. Interestingly, they found that the lightness, chroma and hue weighting factors, which were derived for small colour difference using uniform colour patches under grey backgrounds, needed to be modified for complex images and could be image dependent. This indicates that CIE Colorimetry based colour difference formulae cannot be directly applied to complex images. Further, previous studies actually demonstrated that the conventional approach by averaging each pixel's colour difference often provided an inaccurate representation of the perceived colour difference. Zhang and Wandell considered the situation where a pixel-bypixel averaging of the CIELAB colour differences would certainly fail for half-tone images. They have also evaluated several colour image fidelity metrics and again demonstrated that the predicted visual difference calculated using point-by-point RootMean-Square error in RGB values and CIELAB $\Delta E_{94}$ values all failed to predict the image distortion maps [10] obtained by visual experiment. Therefore they proposed the use of SCIELAB [11], a spatial extension of CIELAB that incorporates spatial low-pass filtering in an opponent colour representation prior to the CIELAB calculation. McCann studied another case, i.e. when all the pixels in an image have a reproduction error in the same direction (lightness, chroma, hue), the colour constancy mechanism of the human visual system would make large errors appear small; when all the errors for each pixel are 
randomly distributed, small errors appear large. Uroz confirmed this phenomenon in his research on colour difference perceptibility for printed images [12]. It seems that the human eyes care more about the relationships of the parts of the image than they do about the absolute value of the match and McCann proposed the use of edge ratios [13] to solve this problem. Lambrecht and Farrell have proposed a metric based on a multi-channel model of human spatial vision and incorporates modeling of colour perception, channel decomposition, contrast sensitivity and visual masking [14]. However, the model was only tested on JPEG-distorted images. This paper describes the study of another more practical phenomenon where each reproduced colour presented in the image has different level of colour difference. For example in cross-media colour image reproduction, rendering images on different devices inevitably introduces colour changes or shifts because of the differences of the colour gamut of different reproduction devices, and it is common that some colours or areas are less accurately reproduced than others because device characterisation usually does not produce the same prediction accuracy for all the colours. This phenomenon becomes even more significant when there is a large colour gamut difference between the original media and the reproduction media and some kind of gamut mapping algorithm is applied. Our experiments will show that the simple pixel averaging method fails to provide an accurate measure of the colour difference for this type of colour image reproduction and our proposed new algorithm is able to solve this problem.

\section{The proposed new algorithm}

Current colour difference formulae are based on colour patches that subtend 4 degrees or more of the viewing field. However, colour patches in images tend to be much smaller. It 
is well known that our eyes tend to be more tolerant towards colour errors of such smaller patches; however, systematic errors over the entire image would be quite noticeable and unacceptable. Hence, for the image-based colour difference algorithm, image analysis is necessary to identify image areas of interest or significance. Therefore, it is not surprising that a simple extension of CIE Colorimetry based colour difference formulae does not work well with complex images. Nonetheless, the validity of these formulae has been extensively tested on solid colour patches and they should be brought into play for calculating the image-based colour difference. Thus, the question remaining is how these formulae should be applied to images. The proposed new algorithm for calculating colour difference between images is based on the following observations made during psychophysical experiments on comparing colour images:

1) The overall colour difference between the images can be calculated as a weighted sum of colour difference between pixels. Since the CIE Colorimetry based colour difference formulae have already been validated for uniform colour patches in numerous occasions, they should serve as a building block towards the image-based colour difference formula. One obvious problem with the conventional method is that every pixel difference is weighted equally. However, not all pixels are equally important when viewing an image. For example, human faces and eyes usually attract much more attention than any other parts of the image do. If pixels or areas of high significance can be identified through image analysis and a suitable weights allocation scheme can be found, major progress can be expected for calculating colour difference for images.

2) Larger areas of the same colour should be weighted higher. This is rather intuitive but is true in most psychophysical experiments. The experiments carried out on comparing 
image difference showed that observers tended to focus on certain areas, usually areas of significant size, of an image, and gave their judgements mainly based on the colour difference of those areas. This assumption agrees with the well-known fact that human eyes tend to be more tolerant towards colour difference of smaller image areas. An extreme example is that it is a very difficult task to notice the colour difference between two pixels. Therefore, it does make sense to assign higher weights to those areas of larger size.

3) Larger colour difference between the pixels should be weighted higher. One shortfall of the current CIE Colorimetry based colour difference formulae is that they are mainly meant for small colour difference only. Thus, they are not capable of giving accurate perceived colour difference for large colour differences. But for an image, it is possible that the reproduced colour of certain pixels or area is quite different from the original, especially when gamut mapping algorithm is applied. The appearance of the whole image usually becomes unacceptable when there are areas of very large colour difference, yet the rest of the image is well reproduced. In the proposed new algorithm, a power function of 2 is adopted to increase the weights allocated to image areas of large colour differences. Several previous studies $[9,12,15]$ suggested that colour difference of $4 \Delta E$ units was probably the acceptability threshold for comparing images. Therefore, this threshold of 4 is built into our image-based colour difference algorithm together with the power function of 2. That means, when the colour difference is bigger than 4 , the weights for the overall colour difference will be increased according to the power function; otherwise, the weights will be reduced. 
4) Hue is an important colour percept for discriminating colours within image context. The hue of an object is normally dictated by the light-absorbing or reflecting properties of the material of which the object is made. The lightness and chroma of the object, however, is seriously affected by illumination and viewing angle. For example, a shadow falling across an object will usually have more effect on the lightness and chroma of the pixels therein than on the hue. Because the proposed new algorithm is based on image histogram analysis, it is therefore necessary to segment the image in the hue plane rather than in three-dimensional colour space to obtain meaningful results. In our algorithm, the full range of CIELAB hue-angle $\left(0^{\circ}\right.$ to $\left.360^{\circ}\right)$ is compressed by half, i.e. the histogram has only 180 different hue-angles.

The following is the proposed new algorithm for image-based colour difference:

I): $\quad$ To transfer each pixel's $L^{*}, a^{*}, b^{*}$ values into $L^{*}, C^{*}{ }_{a b}, h_{a b}$.

II): $\quad$ Calculate the histogram of the $h_{a b}$ image plane, i.e. the probability of each hueangle's occurrences, and store the histogram information in an array hist[hue].

III): The array hist[hue] is sorted in an ascending order. Then this array is divided into 4 sections:

1) For the first $n$ hue-angle in the array hist $\left[\right.$ hue], while $\sum_{i=0}^{n} h i s t[i]<25 \%$, $\operatorname{hist}[i]=h i s t[i] / 4$;

2) For the next $m$ hue-angle in the array hist [hue], while $\sum_{i=n+1}^{n+m} h i s t[i]<25 \%$, hist $[i]=h i s t[i] / 2$; 
3) For the next $l$ hue-angle in the array hist $[$ hue $]$, while $\sum_{i=n+m+1}^{n+m+l} h i s t[i]<25 \%$, hist $[i]=$ hist $[i]$;

4) For the rest of hue-angle in the array hist [hue $]$, hist $[i]=h i s t[i] * 2.25$.

IV): $\quad$ For each hue-angle existed, the average colour difference of all the pixels having that same hue-angle in the image is calculated and stored in $C D[h u e]$.

V): The overall colour difference for the whole image is calculated by $C D_{\text {image }}=\sum$ hist $[\text { hue }]^{*} C D[\text { hue }]^{2} / 4$.

The probability change introduced by the algorithm is arranged in such a way that, for most natural images, the cumulative probability of all hue angles after modification should be very close to 1 , which is the sum of the probabilities of all hue angles in the original image.

\section{$3 \quad$ Results and discussions}

Figures 1(b) and 2(b) show two standard images ("Woman with glass" and "Threads") that were chosen from ISO/DIS 12640-2 [16]. These images are encoded with 8 bits per channel in CIELAB colour space, and the resolution of each image is 400 by 300 pixels. Figure 1(a) is a simulated reproduction in which the same colour difference (a combination of lightness, chroma, and hue shifts) is applied to every pixel in the image. Figure 1(c) is another simulated reproduction in which only the colours of the human skin and the blue dress and decorations are altered (a combination of lightness, chroma, and hue shifts), with the rest of the image unchanged. Similarly, in Figure 2(a) every pixel in the image is 
shifted by the same colour difference. In Figure 2(c), the colour changes are made only to the red and pink bows, the red ribbon, and the red and pink ball of threads. Calculated by averaging each pixel's difference using CIELAB, colour difference between 1(b) and 1(a) ( $\Delta E_{a b}=3.75$ ) is the same as the colour difference between 1(b) and 1(c) ( $\Delta E_{a b}=3.72$ ). In the same way averaging colour difference produces the same colour difference between 2(b) and 2(a) ( $\left.\Delta E_{a b}=3.81\right)$ and between 2(b) and 2(c) $\left(\Delta E_{a b}=3.78\right)$.

An effective image difference metric has to be consistent with the human visual judgements. Therefore, a psychophysical experiment was carried out to find out how human visual systems compare images and judge the difference. Although CIELAB encoding of the images provides the convenience for the mathematical calculations of image colour difference, images encoded in CIELAB cannot be directly displayed on a CRT monitor. These 8-bit CIELAB encoded data was converted to 8-bit RGB data by a CRT characterisation profile generated by a simple gain-offset-gamma (GOG) model [17]. The images were then displayed on a CRT monitor for viewing, and pair comparison was adopted to evaluate the perceived image difference. The experiment was conducted on a Barco monitor in a dark room; a software was created to control the procedure and record the results. Each image displayed was surrounded with a white border which extended about 30 pixels wide and mid-grey was chosen as the background colour. Before the experiment, observers were allowed about 3 minutes to adapt to the viewing condition of the dark room and asked to sit comfortably in front of the monitor about 18 inches away; the following instruction was given: 
In this experiment, you will be shown an original image and two reproductions of it on the monitor at each time. You are asked to select which reproduction has smaller difference with the original. If you really could not determine which one has smaller difference, you can select "SAME” button.

Ten observers participated in the experiment and it was unanimously judged by all observers that Figures 1(c) and 2(c) were having a much larger colour difference than Figures 1(a) and 2(a) respectively. The experimental results show that the simple pixel averaging method fails to provide an accurate measure of the colour difference perceived by human observers.

Table 1 summarises the results obtained by the conventional average method and the proposed new algorithm. The usefulness of the new proposed algorithm lies with the fact that it can "see" the perceptual colour difference that cannot be detected by the conventional pixel-averaging method. Unlike judging colour difference for uniform colour patches, observers tend to find it very difficult to judge colour difference of complex images in terms of numeric values. However, it is a much easier task to judge which reproduction is closer to the original image. Thus, a plausible way for achieving imagebased colour difference is to start with a metric that is capable of ranking a series of reproductions in the same way as human observers would do. It was found that higher weightings for large colour difference occurred in the reproduced image is essential. This finding agrees with Uroz's results that large colour differences of local areas contribute considerably towards overall colour difference. 


\section{Conclusion}

This paper has shown that the conventional average method does not accurately reflect the perceived colour difference of images in many cases. We have proposed a new algorithm, which solves the problem by identifying areas of visual significance within the image and assigning higher weights to those areas. Thus, image differences occurring in the areas of visual significance will contribute more towards the overall colour difference of the whole

image. Being simple and elegant, the new algorithm provides a quantitative method for comparing images that more closely matches a subjective comparison.

\section{References}

1. S. N. Pattanaik, M. D. Fairchild, J. A. Ferwerda, and D. P. Greenberg: 'Multiscale model of adaptation, spatial vision and colour appearance', Proceedings of IS\&T/SID $6^{\text {th }}$ Color Imaging Conference, Scottsdale, USA, November 1998, 2-7.

2. M. D. Fairchild and G. M. Johnson: 'Meet iCAM: a next-generation color appearance model', Proceedings of IS\&T/SID $10^{\text {th }}$ Color Imaging Conference, Scottsdale, USA, November 2002, 33-38.

3. F. H. Imai, N. Tsumura and Y. Miyake: 'Perceptual colour difference metric for complex images based on Mahalanobis distance', Journal of Electronic Imaging, 2001, 10(2), 385-393.

4. T. Newman: 'CIE Division 8: New Directions and Dimensions to Colour Standards', Proceedings of International Colour Management Forum’, Derby, UK, May 1999, University of Derby, 7-14. 
5. 'Colorimetry’, CIE Publication 15.2, CIE Central Bureau, Vienna, 1986.

6. F. J. J. Clarke, R. McDonald, and B. Rigg: 'Modification to the JPC79 colour difference formula', J. Soc. Dyers Col., 1987, 103, 86-94.

7. 'Industrial colour-difference evaluation', CIE Publication 116, CIE Central Bureau, Vienna, 1995.

8. M. R. Luo, G. Cui, and B. Rigg: 'The development of the CIE 2000 colour-difference formula: CIEDE2000’, Colour Res. Appl., 2001, 26(5), 340-350.

9. T. Song and M. R. Luo: 'Testing color-difference formulae on complex images using a CRT monitor', Proceedings of IS\&T/SID $8^{\text {th }}$ Color Imaging Conference, Scottsdale, USA, November 2000, 44-48.

10. X. Zhang and B. A. Wandell: 'Color image fidelity metrics evaluated using image distortion maps’, Signal Processing, 1998, 70(3), 201-214.

11. X. Zhang and B. A. Wandell: 'A spatail extension of CIELAB for digital color image reproduction’, SID Symposium Technical Digest, 1996, 27, 731-734.

12. J. Uroz: 'Colour difference perceptibility for large-size printed images', Proceedings of $8^{\text {th }}$ Colour Image Science Conference, Scottsdale, USA, November 2000, 138-151.

13. J. J. McCann: 'Color imaging systems: past, present and future', Proceedings of $5^{\text {th }}$ International Conference on high technology: imaging science and technology, Chiba, Japan, May 1996, 2-12.

14. C. J. Lambrecht and J. E. Farrell: 'Perceptual quality metric for digitally coded color images’, Proceedings of the European Signal Processing Conference, Trieste, Italy, September 1996, 1175-1178. 
15. M. Stokes: 'Colorimetric tolerances of digital images', MSc Dissertation, Rochester Institute of Technology, USA, 1991.

16. 'Graphic technology - Prepress digital data exchange - XYZ/sRGB standard colour image data (XYZ/SCID)', ISO standard 12640-2, 2000.

17. R. S. Berns: 'Methods for characterizing CRT displays', Displays, 16(4), 1996,173182. 


\begin{tabular}{l|l|l|l|l}
\hline \multirow{2}{*}{ Colour difference formula } & \multicolumn{3}{|c|}{ Woman with glass } & \multicolumn{2}{c}{ Threads } \\
\cline { 2 - 5 } for images & (b) and (a) & (b) and (c) & (b) and (a) & (b) and (c) \\
\hline Conventional average & 3.75 & 3.72 & 3.81 & 3.78 \\
Proposed new algorithm & 3.60 & 8.98 & 3.92 & 9.95 \\
\hline
\end{tabular}

Table 1 Experimental results obtained 